\title{
Applicability of Ionic Liquids in Topical Drug Delivery Systems: A Mini Review
}

\author{
Rita Caparica, Ana Júlio, Joana Portugal Mota, Catarina Rosadoand Tânia Santos de Almeida* \\ CBIOS-Research Center for Biosciences and Health Technologies, Lusófona University, Portugal
}

Submission: December 20, 2017; Published: January 04, 2018

*Corresponding author: Tânia Santos de Almeida, CBIOS-Research Center for Biosciences and Health Technologies, Lusófona University, Campo Grande 376, 1749-024 Lisbon, Portugal; Email: tania.almeida@ulusofona.pt

\begin{abstract}
The Pharmaceutical Industry has several challenges to overcome, such as poor drug solubility, skin permeation, problems related with the stability of the prepared delivery systems, as well as the type of administration to be used. When choosing the most suitable route of administration, non-invasive routes of administration are preferably chosen, with the oral administration being one of the most used. However, the delivery of drugs through the skin can be an alternative route of administration that avoids the gastrointestinal tract, and overcomes complications associated with the parenteral route since it is a non-invasive technique and is widely accepted among patients. Nonetheless, when formulating these delivery systems other problems need to be addressed, such as the low solubility of many drugs and the reduced stability of some of the developed systems. Thus, finding functional excipients that help to overcome these problems is fundamental.
\end{abstract}

In this context, the valuable properties of ionic liquids will be reviewed herein, particularly with respect to their application in the pharmaceutical field and in topical delivery. Furthermore, some studies, that show the value of ionic liquids as drug solubility/loading, permeation enhancers in topical drug delivery systems, will be summarized.

Keywords: Drug Delivery Systems; Ionic Liquids; Topical Drug Delivery; Solubility; Permeation; Toxicity; Functional Ingredients

Abbreviations: \%: Percentage; [Bmim][Br]: 1-Butyl-3-methylimidazolium bromide; [Bmim][C12SO3]: 1-Butyl-3-methylimidazolium dodecanesulfate; [Bmim][PF6]: 1-Butyl-3-methylimidazolium hexafluorophosphate; [C1mim][(CH3O2)PO2]: dimethylimidazoliumdimethylphosphate; [C2mim][Br]: 1-Ethyl-3-methylimidazolium bromide; [C4mim][Br]: 1-Ethyl-3-butylimidazolium bromide; [C6mim][Br]: 1-Ethyl-3-hexylimidazolium bromide; [CDHP]:Cholinedihydrogenphosphate; [Cho][Glu]: (2-Hydroxyethyl) trimethylammoniumglutamate; [Cho][Phe]: (2-Hydroxyethyl) trimethylammoniumL-phenylalaninate; [Emim][EtSO4]: 1-Ethyl-3-methylimidazolium ethyl sulfate; [Hmim][Cl]: 1-Hexyl-3-methylimidazolium chloride; [HOEIM][Cl]: 1-Hydroxyethyl-3-methylimidazolium chloride; [HPyr][Cl]: Hexylpyridinium chloride; DMBA: Dimethylbenz(a)anthracene; ETO: Etodolac; IL/o: Ionic liquid-in-oil; IL/w: Ionic liquid-in-water; ILs: Ionic Liquids; IPM: Isopropyl myristrate; m2: Square metre; ME: Microemulsion; nm: Nanometers; O/W: Oil-in-water; RTILs: Room Temperature Ionic Liquids; SEPINEO ${ }^{\text {TM }}$ P600: Acrylamide/sodium acryloyldimethyltaurate copolymer/isohexadecane / polysorbate 80; Span 20: Sorbitan laurate; TPA: 12-0-tetradecanoylphorbol-13-acetate; Tween 80: Polyoxyethylenesorbitanmonooleate; W/0: Waterin-oil

\section{Introduction}

Nowadays, several possibilities for drug administration are already known and widely used. The choice of the most suitable route of administration to use depends on factors such as the drug to be used, its pharmacokinetic profile, as well as the desired target site $[1,2]$.Furthermore, the choice of route of administration to be used also depends on other factors such as its invasiveness [2]. Thus, non-invasive routes of administration are preferably chosen [2]. And the oral administration is one of the most used [3]. However, despite the advantages inherent in oral and even parenteral use, the pharmaceutical industry has been encouraged to explore alternative administration routes that also allow efficient and effective drug delivery [4].
The delivery of drugs through the skin is widely accepted among patients [5]. Can be an alternative route of administration that avoids the gastrointestinal tract, and overcomes complications associated with the parenteral route since it is a non-invasive technique [2]. Another drawback that the pharmaceutical Industry faces when developing drug delivery systems relates to the low solubility of many drugs as well to the reduced stability of those systems. Thus, finding functional excipients that help to overcome these problems is fundamental. Recently, some studies have shown the relevance of using ionic liquids (ILs) to improve drug solubility/loading, permeation of topical drug delivery systems [6-12]. 


\section{Drug Delivery through the Skin}

Delivery through the skin can be divided into two classes: dermal/topical delivery and transdermal delivery. Absorption of drugs through the skin occurs in both delivery systems although the local where the drug is intended to produce effect is different. In the transdermal delivery it is intended that the drug, applied to the skin, reaches the blood circulation to produce a systemic therapeutic effect $[2,3,13]$. On the other hand, in the topical delivery the target site is within the skin itself, with minimal or without systemic absorptions. This system is generally used for the treatment of local diseases such as dermatological treatments, e.g. psoriasis, acne, fungal infections $[14,15]$ and/ or contact dermatitis [16]. Moreover, the topical drug delivery system can still be applied to healthy skin through cosmetic formulations [15]. There are several types of formulations capable of topically delivering drugs and the most commonly used are solutions, emulsions, suspensions, semisolids, sprays and solids [15].

\section{Topical Drug Delivery Systems}

The topical drug delivery, comparatively with other routes, mainly with the oral route, avoids the first pass metabolism and other inconveniences such as $\mathrm{pH}$, presence of enzymes and gastric emptying time associated to the gastrointestinal tract $[3,15,17]$. Furthermore, topical administration has more advantages such as: large area of application in comparison with buccal or nasal cavity, improved patient compliance and acceptance, ease and convenience of application, suitability for self-medication, painless and non-invasive administration, allowing the use of drugs with narrow therapeutic window and short biological half-life, ease of dose termination in the event of any adverse reactions and allows direct access to target site and consequently a more specific drug delivery, providing an alternative in circumstances where oral dosing is not possible $[3,17,18]$.

However, there are known disadvantages associated with the use of topical delivery systems for drugs, some of which are related to the drug, such as poor drug solubility/loading to be used and others with the characteristics and properties of the skin, that may lead to skin irritation, due to the drug and excipients, difficulty in the absorption of drugs with a high particle size, poor permeability of some drugs, possible denaturation of drugs by the enzymes in the epidermis and possible occurrence of allergic reactions [17]. Thus, considering some of these disadvantages, for the development of formulations for topical application it is fundamental to know and understand the characteristics and properties of the skin and of the drugs to incorporate in the developed topical systems.

\section{The Skin barrier}

The skin is the largest and one of the most important organs of the human body, covering about $1.7 \mathrm{~m} 2$ and representing approximately $10 \%$ of the total body mass of an adult [19]. This structure has several functions but its primary function is to provide an effective barrier between the human body and the external environment by protecting it against external factors that can induce damages, such as chemicals, ultraviolet radiation, allergens and microorganisms and the loss of water and nutrients $[19,13]$. Human skin is composed of the appendages, eccrine and apocrine sweat glands and hair follicles, and by three different main layers: the epidermid is, the dermis and the hypodermis [13]. The stractumcorneum, often referred as non-viable epidermis, is the outmost layer and primary barrier of the skin due to its unique structure consisting of layers of flattened corneocytes surrounded by lipid bilayers composed mainly of ceramides [20]. Most of the compounds applied topically penetrate the skin through the intercellular route, by passing the lipid bilayers of the stratum corneum, although, in some circumstances, they also penetrate across the transcellular route [20]. Therefore, since certain layers of the skin may limit the bioavailability of some drugs, a careful selection of the drugs is essential as well as of the excipients used and a clear understanding of their properties to develop formulations for topical drug delivery.

\section{Ionic Liquids}

Skin has a hydrophilic and a hydrophobic domain and consequently the drug usedneeds to have the ability to permeate both domains [19]. However, poor drug solubility of many drugs in water and in most pharmaceutical grade solvents, used in the developed topical formulations, represents an obstacle. Hence, several efforts have been made to find ingredients that may assist in this challenge, and ionic liquids have been used for this purpose [6-12]. ILs are salts, with an organic cation and an inorganic or organic [21-23], that are liquid at temperatures below $100{ }^{\circ} \mathrm{C}$ or even at room temperature [21,24,25]. These latter are known as Room Temperature Ionic Liquids (RTILs).

They have numerous prised characteristics such as low vapour pressures [21,26], possibility of recycling [27], negligible volatile [28], non flammability [10,26,27], high ionic conductivity [29], high thermal and chemical stability [29] and also their ability to dissolve inorganic, organic and polymeric materials, which becomes extremely relevant when using these salts has ingredients in delivery systems [21]. Another extremely important property of ILs is their high suitability for alterations, since this allows their properties to be tailored accordingly to a specific application, by intentionally modifying their anions and/or cations, $[26,27,29,30]$. In terms of their categorisation, ILs have been generally classified in three generations (Figure 1 ), according the structure and chemical properties of the ILs [9,31]. The first generation of ILs are composed by the dialkylimidazolium and alkylpyridinium cations with metal halide anions and although they have valuable properties they are air- and water-sensitive. 


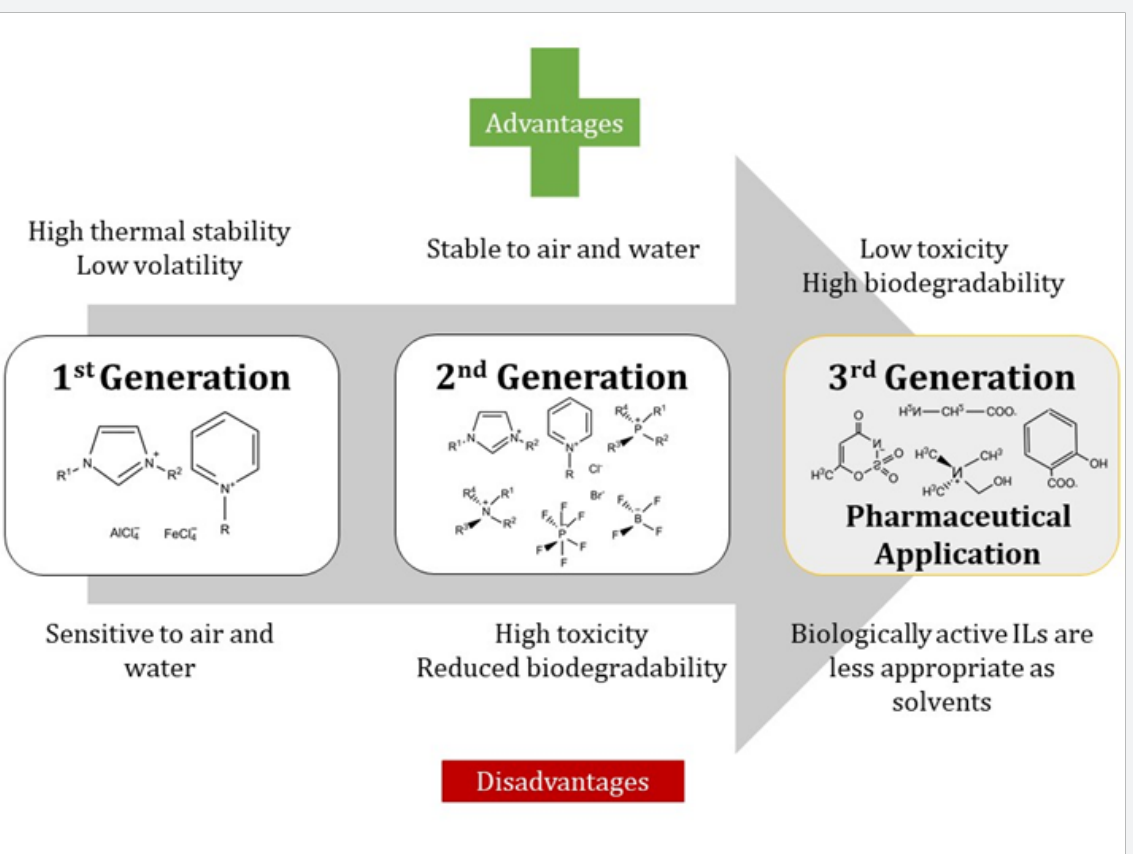

Figure 1: Generation of lonic Liquids: the evolution through time focusing their properties, advantages and disadvantages.

On the other hand, the second generation of ILs are known to have the advantage to be stable to air and water and are normally formed by the dialkylimidazolium, alkylpyridinium, ammonium and phosphonium cations and the tetrafluoroborate and hexafluorophosphat anions. Finally, the third generation is constituted by biodegradable and natural ions or by ions with known biological activities and choline-based ILs with amino acids are amongst the most usual ILs in this last generation [9,31]. Hence, this last generation of ILsare very exciting materials to work with due to their possible applications in pharmaceutics, ecology and/or biology fields and choline-based ILs with amino acids are the most usual ILs in this last generation [9,31]. ILs have also been classified into four main categories, according the cation that constitutes them, either dialkylimidazolium, N-alkylpyridinium, phosphonium or a alkylammonium cation $[11,27]$.

The most studied IL are the imidazolium-based, since they are stable, within reductive and oxidative conditions and they have a lower viscosity and an easier synthesis $[11,27]$. However, the they have been described as being toxic, with their toxicity dependent on the size of the alkyl chain which limits their applicability, particularly in the pharmaceutical field [11,32,33]. Nonetheless, these ILs have been proven useful as solvents, catalystsor even as solubility promoters $[11,21]$. Thepyridiniumbased ILs, have also been used as solvents $[11,27,34]$ and catalysts [35] in several organic reactions and even in the synthesis of pharmaceutical compounds [36].

On the other hand, although phosphonium-based ILs have also been used as catalysts and solvents in several reactions $[37,38]$ they have higher thermal stability than imidazolium- and ammonium-based salts, and thus are more suitable for reactions at temperatures above $100{ }^{\circ} \mathrm{C}$ [39]. Regarding the more recent quaternary ammonium-based ILs, , they have been described as the less toxic class of ILs $[22,40]$ and consequently have been described in the literature as "green" alternatives to the solvents normally used in the pharmaceutical industry [40]. It becomes clear that ILs have been broadly used as solvents, catalysts and reaction media in several reactions [26] and their possible applicability in the health area is very appealing. None the less, the toxicity of this class of compounds needs to be carefully considered.

\section{ILs in Topical Drug Delivery Systems}

As mentioned previously, ILs may be tailored to be incorporated in different types of mediums such as water, oils or hydro alcoholic solutions, depending upon the ion present, which allows them to be included in different drug delivery systems, such as topical systems. One of the earliest works, that studied the incorporation of ionic liquids in this topical delivery systems, was done by Dobler and co-workers [21]. In this study, oil-in-water $(\mathrm{O} / \mathrm{W})$ and water-in-oil $(\mathrm{W} / \mathrm{O})$ emulsions were prepared with two different ILs, $[\mathrm{Hmim}][\mathrm{Cl}]$ and $[\mathrm{Bmim}][\mathrm{PF} 6]$, to replace the water and oily phases, respectively andboth ILs were successfully incorporated into the stable emulsions. These emulsions showed antimicrobial activity in a range above $5 \%$ of IL, and a higher skin penetration, especially the formulation containing [Bmim][PF6], the lipophilic IL.After this work, the same group investigated the influence of the ILs, [HPyr][Cl], [CDHP] and [Emim][EtSO4], in drug skin penetration and their antimicrobial properties in an emulsion-gel of 4-hydroxybenzoic 
acid propyl ester, caffeine and testosterone [11]. In terms of skin penetration of the hydrophilic drug, caffeine, after $24 \mathrm{~h}$, this parameter was two-fold higher when compared with the formulation in the absence of [HPyr][Cl]. While the testosterone, the lipophilic drug, did not observe any modifications in the skin penetration. Furthermore, the addition of imidazoliumand pyridinium-based ILs reduced strongly the viscosity of the emulsion-gel with SEPINEO ${ }^{\mathrm{TM}}$ P600, withoutmodifying the rheology of thehydroethylcellulose gel. It was also showed that over storage time (3 months), all formulations were stable. Thus, results indicate that the studied ILs may be useful to enhance skin penetration in topical formulations.

Furthermore, ILs have also been studied in a minor scale, through the preparation of ionic liquid-in-oil (IL/o) microemulsions (ME) as drug carriersof poorly aqueous soluble drugs, such as acyclovir, methotrexate and 1-[(5-(p-nitrophenyl) furfurylidene) amino] hydantoin sodium (dantrolene sodium) [29,30]. In these studies, Moniruzzaman and co-workersincorporated nanometer-sized ILs droplets in isopropyl myristrate (IPM) with a blend of Tween80 and Span20, with the ILs as a dispersed phase. This study demonstrated a highersolubilisation ofstudied drugs in the ILs ME. These studies also showed that the percentage of ILs used in the ME strongly affects the toxicity of the system, and although with $4 \%$ of IL, $80 \%$ of cell viability is maintained, pure IL leads to a significant decrease in cell viability.

Another IL/o ME with 5-fluorouracil (5-FU) was developed using IPM, Tween $80 /$ Span 20 and [Bmim][Br] as IL [41]. In this study, the solubility of 5-FU was also enhanced with results showing a solubility 2.6 times higher in the presence of the IL than in aqueous solution. On the other hand, small and mono disperse droplets were formed and ex vivo permeation studies in animal models also showed an increase of this parameter for 5-FU when compared to aqueous solution and W/O ME. Additionally, in vivo studies using dimethylbenz(a)anthracene (DMBA)/12-0-tetradecanoylphorbol-13-acetate (TPA)-induced mice skin carcinogenesis model proved that erythema and irritation was not observed as side effects, that results were supported by the histopathological analysis.

Another study prepared an ionic liquid-in-water (IL/w) ME with Etodolac (ETO) containing [Bmim][PF6], Tween 80 and ethanol [42]. Ex vivo permeation studies using rat skin and it showed that the IL/w ME had a higher ETO permeation comparing with the oily solution of ETO and to the O/W ME. Also, IL containing system was more effective in controlling in vivo inflammation than the oily solution or $\mathrm{O} / \mathrm{W}$ ME or the sold formulation of ETO. More recently, some studies have been done comparing imidazole-based and choline-based ILs, both classes used at non-toxic concentrations, to show their ability as functional ingredients in topical delivery systems [11,33]. In these study, three imidazole-based ILs, [C2mim][Br], [C4mim]
$[\mathrm{Br}]$ and [C6mim][Br] and two choline-based ILs, [Cho][Phe] and [Cho][Glu] were studied and since incorporation in topical formulations was sought, their cytotoxicity was characterized in human keratinocytes (HaCat cells). Furthermore, caffeine and salicylic acid were used as model actives and their solubility and permeation through pig ear skin, in the presence and absence of the studied ILs, was evaluated. Results showed that at non-toxic concentrations, were cell viability was maintained, the cholinebased ILs were more suited as functional ingredients, since they allowed a higher enhancement in drug solubility, particularly for caffeine. Furthermore, results also showed that [Cho][Phe] and [Cho][Glu], do not impact drug permeation which may be relevant to ensure low incidence of adverse effects in topical formulations where low drug permeation is pursued.On the other hand, the imidazole-based ILs proved to be more toxic, with their toxicity enhancing with the size of the alkyl chain, and when used at non-toxic concentrations, their utility as solubility promoters was less evident. Furthermore, the authors also evaluated the incorporation of caffeine into $0 / \mathrm{W}$ emulsions and gels using the two choline-based ILs at non-cytotoxic concentrations, and all formulations were stable after stress stability studies, showing that these ILs did not influence the rheology of $\mathrm{O} / \mathrm{W}$ emulsions and gels. Hence, Choline and amino acids based ILs that have been described as less toxic and more enviromently-friends [22], show the promising ability to be used as functional ingredients in topical delivery systems.

More recently, two imidazolium-based ILs, [HOEIM] [Cl] and [Bmim][C12SO3], were also incorporated into ME with Dencichine [43]. Results of the in vitro skin permeation proposed a 10 -fold improvement for the formulation with ILs, because the nano carrier with ILs disrupted the regular skin barrier properties and reorganised the corneocytes that modify the stratum corneum surface properties. Furthermore, the in vivo pharmacodynamic analysis demonstrated an important haemostatic activity of Dencichine by topical application, as well as, a reduced cell toxicity and skin irritation. These results show once again, that ILs may have multiple utilities when incorporated in topical Delivery systems [44-63].

\section{Conclusion}

This mini-review shows the valuable properties of Ionic Liquids and their potential applicability in the Pharmaceutical and Medical fields, particularly as functional ingredients in topical drug delivery systems. The structure and functional flexibility of ILs may be useful for their incorporation in different mediums, allowing them to be included indifferent topical drug delivery systems, and improving their performance. Currently, there are some studies that already show this ability of ionic liquids to work as performance enhancers of topical delivery systems. Nonetheless, the characteristics of the ILs used are truly relevant for their efficacy, particularly when considering their toxicity, which may be a limitative characteristic. In fact, 
for these salts truly act as functional ingredients, they need to be functional at non-toxic concentrations were cell viability is maintained. In this context, recent studies have already shown that some ILs may be more suited as functional ingredients, in terms of their toxicity. Furthermore, the interaction between ILs and other excipients or functional ingredients needs also to be considered.

Nonetheless, ILs have the remarkable ability to be tailored accordingly to a desired goal, and consequently, much is still to be done in this area to encounter new ILs that may be truly useful in enhancing the performance of a topical delivery system.

\section{Acknowledgment}

The authors would like to thank to FCT, Portugal (UID/ DTP/04567/2016).

\section{References}

1. Brocks DR, Mehvar R (2010) Rate and extent of drug accumulation after multiple dosing revisited. Clinical Pharmacokinetics 49(7): 421438.

2. Torin Huzil J, Sivaloganathan S, Kohandel M, Foldvari M (2011) Drug delivery through the skin: Molecular simulations of barrier lipids to design more effective noninvasive dermal and transdermal delivery systems for small molecules, biologics, and cosmetics. Wiley Interdisciplinary Reviews: Nanomedicine and Nanobiotechnology 3(5): 449-462.

3. Brown MB, Martin GP, Jones S, Akomeah FK (2006) Dermal and transdermal drug delivery systems: current and future prospects. Drug Delivery 13(3): 175-187.

4. Singh Malik D, Mital N, Kaur G (2016) Topical drug delivery systems: a patent review. Expert Opinion on Therapeutic Patents 26(2): 213-228.

5. Kumar S, Singh N, Arora SC (2015) Emulgel an Insight. European Journal of Pharmaceutical and Medical Research 2(4): 693-698.

6. Dobler D, Schmidts T, Zinecker C, Schlupp P, Schäfer J, et al. (2015) Hydrophilic Ionic Liquids as Ingredients of Gel-Based Dermal Formulations. AAPS Pharm Sci Tech 17(4): 923-931.

7. Frade RF, Afonso CA (2010) Impact of ionic liquids in environment and humans: an overview. Human and Experimental Toxicology 29(12): 1038-1054.

8. Frizzo CP, Gindri IM, Tier AZ, Buriol L, Moreira DN, et al. (2013) Pharmaceutical Salts : Solids to Liquids by Using Ionic Liquid Design. In Ionic Liquids-New Aspects for the Future pp. 557-580.

9. Hough WL, Smiglak M, Rodríguez H, Swatloski RP, Spear SK, et al. (2007) The third evolution of ionic liquids: active pharmaceutical ingredients. New Journal of Chemistry 31(8): 1429.

10. Mizuuchi H, Jaitely V, Murdan S, Florence AT (2008) Room temperature ionic liquids and their mixtures: Potential pharmaceutical solvents. European Journal of Pharmaceutical Sciences 33(4-5): 326-331.

11. Santos De Almeida T, Júlio A, Saraiva N, Fernandes AS, Araújo MEM, et al. (2017) Choline- versus imidazole-based ionic liquids as functional ingredients in topical delivery systems: cytotoxicity, solubility, and skin permeation studies. Drug Development and Industrial Pharmacy 43(11): 1858-1865.

12. Stoimenovski J, MacFarlane DR, Bica K, Rogers RD (2010) Crystalline vs. ionic liquid salt forms of active pharmaceutical ingredients: A position paper. Pharmaceutical Research 27(4): 521-526.
13. Gabriel T (2016) Topical Antiacne Drugs Delivery Systems. The Open Dermatology Journal 10(1): 85-95.

14. Joseph J, Daisy PA, George BJR, Thomas N, Carla B (2017) Emulgel: A novel trend in Topical Drug Delivery System. World Journal of Pharmaceutical and Medical Research 3(4): 35-39.

15. Vaishali Arora, Praveen Kumar RS (2015) Emulgels: A Review for Topical Drug Delivery of Hydrophobic Drugs. International Journal of Pharma Professional's Research 6(3): 1256-1263.

16.Zheng Zhanga, Pei-Chin Tsaib, Tannaz Ramezanlib, BB MK (2014) Polymeric nanoparticles-based topical delivery systems for the treatment of dermatological diseases. Wiley Interdisciplinary Reviews: Nanomedicine and Nanobiotechnology 11(3): 393-407.

17. Kaur J, Kaur J, Jaiswal S, Gupta G (2016) Recent Advances in Topical Drug Delivery System. Pharmaceutical Research 6(7).

18. Kute SB, Saudagar RB (2013) Emulsified gel A Novel approach for delivery of hydrophobic drugs: An overview. Journal of Advanced Pharmacy Education \& Research 3(4): 368-376.

19. Heylings JR (2011) Transdermal and Topical Drug Delivery. Transdermal and Topical Drug Delivery: Principles and Practice.

20. Nastiti CMRR, Ponto T, Abd E, Grice JE, Benson HAE, Roberts MS (2017) Topical nano and microemulsions for skin delivery. Pharmaceutics 9(4): 1-25.

21. Dobler D, Schmidts T, Klingenhoefer I, Runkel F (2012) Ionic liquids as ingredients in topical drug delivery systems. International Journal of Pharmaceutics 441(1-2): 620-627.

22. Gouveia W, Jorge TF, Martins S, Meireles M, Carolino M, et al. (2014) Toxicity of ionic liquids prepared from biomaterials. Chemosphere 104: 51-56.

23. Torimoto T, Tsuda T, Okazaki KI, Kuwabata S (2010) New frontiers in materials science opened by ionic liquids. Advanced Materials 22(11): 1196-1221.

24. Ferraz R, Branco LC, Prudêncio C, Noronha JP, Petrovski Ž (2011) Ionic liquids as active pharmaceutical ingredients. Chem Med Chem 6(6): 975-985.

25. Kubota K, Shibata A, Yamaguchi T (2016) the molecular assembly of the ionic liquid/aliphatic carboxylic acid/aliphatic amine as effective and safety transdermal permeation enhancers. European Journal of Pharmaceutical Sciences 86: 75-83.

26. Balk A, Holzgrabe U, Meinel L (2015) Pro et contra' ionic liquid drugs - Challenges and opportunities for pharmaceutical translation. European Journal of Pharmaceutics and Biopharmaceutics 94: 291304.

27. Ghandi K (2014) A Review of Ionic Liquids, Their Limits and Applications. Green and Sustainable Chemistry 4: 44-53.

28. Earle MJ, Esperança JMSS, Gilea MA, Canongia Lopes JN, Rebelo, et al. (2006) The distillation and volatility of ionic liquids. Nature 439(7078): 831-834.

29. Moniruzzaman M, Kamiya N, Goto M (2010) Ionic liquid based microemulsion with pharmaceutically accepted components: Formulation and potential applications. Journal of Colloid and Interface Science 352(1): 136-142.

30. Moniruzzaman M, Tamura M, Tahara Y, Kamiya N, Goto M (2010) Ionic liquid-in-oil microemulsion as a potential carrier of sparingly soluble drug: Characterization and cytotoxicity evaluation. International Journal of Pharmaceutics 400(1-2): 243-250.

31. Egorova KS, Gordeev EG, Ananikov VP (2017) Biological Activity of Ionic Liquids and Their Application in Pharmaceutics and Medicine. Chemical Reviews, A-BF. 
32. Romero A, Santos A, Tojo J, Rodríguez A (2008) Toxicity and biodegradability of imidazolium ionic liquids. Journal of Hazardous Materials 151(1): 268-273.

33. Santos de Almeida T, Júlio A, Caparica R, Rosado C, Fernandes AS, et al. (2015) Ionic liquids as solubility/permeation enhancers for topical formulations: Skin permeation and cytotoxicity characterization. Toxicology Letters.

34. Snelders DJM, Dyson PJ (2011) Efficient synthesis of $\beta$-chlorovinylketones from acetylene in chloroaluminate ionic liquids. Organic Letters 13(15): 4048-4051.

35. Tharun J, Kathalikkattil AC, Roshan R, Kang DH, Woo HC, et al. (2014) Microwave-assisted, rapid cycloaddition of allyl glycidyl ether and CO 2 by employing pyridinium-based ionic liquid catalysts. Catalysis Communications 54: 31-34.

36. Pajuste K, Plotniece A, Kore K, Intenberga L, Cekavicus B, et al. (2011) Use of pyridinium ionic liquids as catalysts for the synthesis of 3,5-bis(dodecyloxycarbonyl)-1,4-dihydropyridine derivative. Open Chemistry 9(1): 143-148.

37. Cao SW, Zhu YJ (2009) Iron oxide hollow spheres: Microwavehydrothermal ionic liquid preparation, formation mechanism, crystal phase and morphology control and properties. Acta Materialia 57(7): 2154-2165.

38. Fan A, Chuah GK, Jaenicke S (2012) Phosphonium ionic liquids as highly thermal stable and efficient phase transfer catalysts for solidliquid Halex reactions. Catalysis Today 198(1): 300-304.

39. Dake SA, Kulkarni RS, Kadam VN, Modani SS, Bhale JJ, et al. (2009) Phosphonium Ionic Liquid - A Novel Catalyst for Benzyl Halide Oxidation. Synthetic Communications: An International Journal for Rapid Communication of Synthetic Organic Chemistry 39(21): 38983904.

40. Melo CI, Bogel-Łukasik R, Nunes da Ponte M, Bogel-Łukasik E (2013) Ammonium ionic liquids as green solvents for drugs. Fluid Phase Equilibria 338(3): 209-216.

41. Goindi S, Arora P, Kumar N, Puri A (2014) Development of Novel Ionic Liquid-Based Microemulsion Formulation for Dermal Delivery of 5-Fluorouracil. AAPS Pharma Sci Tech 15(4): 810-821.

42. Goindi S, Kaur R, Kaur R (2015) An ionic liquid-in-water microemulsion as a potential carrier for topical delivery of poorly water soluble drug: Development, ex-vivo and in-vivo evaluation. International Journal of Pharmaceutics (Vol. 495). Elsevier B.V.

43. Wang C, Zhu J, Zhang D, Yang Y, Zheng L, et al. (2018) Ionic liquid microemulsions assisting in the transdermal delivery of Dencichine: Preparation, in-vitro and in-vivo evaluations, and investigation of the permeation mechanism. International Journal of Pharmaceutics, 535(1-2): 120-131.

44. Álvarez MS, Esperança JMSS, Deive FJ, Sanromán MÁ, Rodríguez A (2015) A biocompatible stepping stone for the removal of emerging contaminants. Separation and Purification Technology 153: 91-98.

45. Chen X, Liu J, Wang J (2010) Ionic liquids in the assay of proteins. Analytical Methods 2(9): 1222.

46. Constatinescu D, Herrmann C, Weigärtner H (2010) Patterns of protein unfolding and protein aggregation in ionic liquids. Physical Chemistry Chemical Physics 12(8): 1756-1763.
47. Czekanski L, Santos de Almeida T, Portugal Mota J, Rijo P, Araújo MEM (2014) Synthesis of benzoazole ionic liquids and evaluation of their antimicrobial activity. Biomedical and Biopharmaceutical Research 11(2): 227-235.

48. Forte A, Melo CI, Bogel-Łukasik R, Bogel-Łukasik E (2012) A favourable solubility of isoniazid, an antitubercular antibiotic drug, in alternative solvents. Fluid Phase Equilibria 318: 89-95.

49. Fujita K, Mac Farlane DR, Forsyth M (2005) Protein solubilising and stabilising ionic liquids. Chemical Communications 70(38): 4804.

50. Gabriel T (2016) Topical Antiacne Drugs Delivery Systems. The Open Dermatology Journal 10(1): 85-95.

51. Ghosh S, Parui S, Jana B, Bhattacharyya K (2015) Ionic liquid induced dehydration and domain closure in lysozyme: FCS and MD simulation. Journal of Chemical Physics 143(12).

52. Handy ST (2011) Ionic Liquids - Classes and Properties.

53. Khupse ND, Kumar A (2011) The cosolvent-directed Diels-Alder reaction in ionic liquids. Journal of Physical Chemistry A 115(36): 10211-10217.

54. Marrucho IM, Branco LC, Rebelo LPN (2014) Ionic liquids in pharmaceutical applications. Annual Review of Chemical and Biomolecular Engineering 5: 527-546.

55. Micaêlo NM, Soares CM (2008) Protein structure and dynamics in ionic liquids. Insights from molecular dynamics simulation studies. The Journal of Physical Chemistry 112: 2566-2572.

56. Moriel P, García-Suárez EJ, Martínez M, García AB, Montes-Morán MA, et al. (2010) Synthesis, characterization, and catalytic activity of ionic liquids based on biosources. Tetrahedron Letters 51(37): 4877-4881.

57. Nandi S, Parui S, Halder R, Jana B, Bhattacharyya K (2017) Interaction of proteins with ionic liquid, alcohol and DMSO and in situ generation of gold nano-clusters in a cell. Biophys Rev.

58. Pernak J, Smiglak M, Griffin ST, Hough WL, Wilson TB, et al. (2006) Long alkyl chain quaternary ammonium-based ionic liquids and potential applications. Green Chemistry 8(9): 798.

59. Rantwijk F Van, Sheldon RA (2007) Biocatalysis in Ionic Liquids Biocatalysis in Ionic Liquids. Chem Rev 107(6): 2757-2785.

60. Santos AD Dos, Morais ARC, Melo C, Bogel-Łukasik R, Bogel-Łukasik E (2013) Solubility of pharmaceutical compounds in ionic liquids. Fluid Phase Equilibria 356: 18-29.

61. Thuy Pham TP, Cho CW, Yun YS (2010) Environmental fate and toxicity of ionic liquids: A review. Water Research 44(2): 352-372.

62. Zakrewsky M, Lovejoy KS, Kern TL, Miller TE, Le V, et al. (2014) Ionic liquids as a class of materials for transdermal delivery and pathogen neutralization. Proceedings of the National Academy of Sciences 111(37): 13313-13318.

63. Zhang D, Wang H, Cui X, Wang C, Zhang D, et al. (2016) Evaluations of imidazolium ionic liquids as novel skin permeation enhancers for drug transdermal delivery. Pharmaceutical Development and Technology 7450(1): 1-10. 


\section{Your next submission with Juniper Publishers will reach you the below assets}

- Quality Editorial service

- Swift Peer Review

- Reprints availability

- E-prints Service

- Manuscript Podcast for convenient understanding

- Global attainment for your research

- Manuscript accessibility in different formats ( Pdf, E-pub, Full Text, Audio)

- Unceasing customer service

Track the below URL for one-step submission https://juniperpublishers.com/online-submission.php 